\title{
Global Registration of Multiple Point Sets: Feasibility and Applications in Multi-fragment Fracture Fixation
}

\author{
Mehdi Hedjazi Moghari ${ }^{1}$ and Purang Abolmaesumi ${ }^{1,2}$ \\ ${ }^{1}$ Department of Electrical and Computer Engineering, Queen's University, Canada \\ 2 School of Computing, Queen's University, Canada \\ purang@cs.queensu.ca
}

\begin{abstract}
An algorithm to globally register multiple 3D data sets (point sets) within a general reference frame is proposed. The algorithm uses the Unscented Kalman Filter algorithm to simultaneously compute the registration transformations that map the data sets together, and to calculate the variances of the registration parameters. The data sets are either randomly generated, or collected from a set of fractured bone phantoms using Computed Tomography (CT) images. The algorithm robustly converges for isotropic Gaussian noise that could have perturbed the point coordinates in the data sets. It is also computationally efficient, and enables real-time global registration of multiple data sets, with applications in computer-assisted orthopaedic trauma surgery.
\end{abstract}

\section{Introduction}

Global registration of multiple 3D data sets is an essential and complex problem in medical imaging and computer vision, where three or more data sets must be aligned in a common reference frame. One of the applications of such registration is in computer-assisted orthopedic trauma surgery, where surgeons are interested in reassembling the bone fractures back into the original solid bone (Figure 1).

Generally the registration problem is divided into two sub-problems of finding corresponding points among data sets, and deriving the transformation parameters that minimize the distance among the estimated correspondences. In this article, we concentrate on the registration task and assume that the corresponding points are either available or the data sets are close enough such that the closest points among the data sets are reliable candidates for the corresponding points. In real applications, the corresponding points are not precisely localized and therefore, they are perturbed by some error (noise), which could be caused by for example, sampling, image segmentation and reconstruction errors. The existence of noise in the data sets is another issue that must be considered in registration algorithms. The statistical property of the noise is usually unknown; however, if it is identified, it can be incorporated in the registration process to improve the accuracy. Here, we assume that the data sets are corrupted by isotropic Gaussian noise with a distribution that may be different for each data set. 


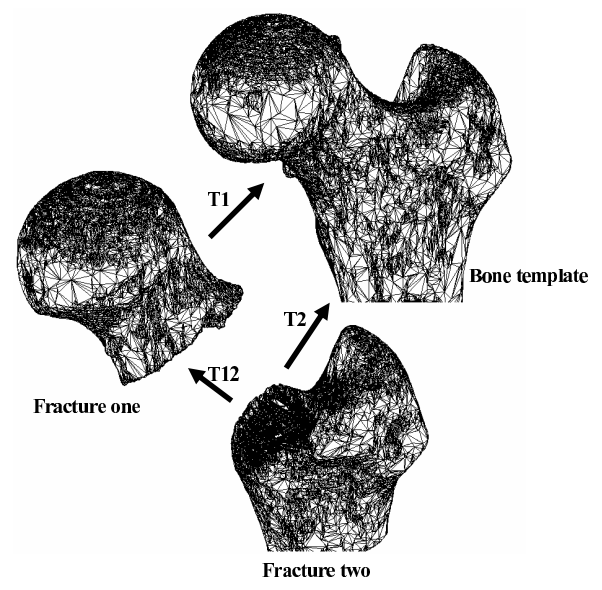

Fig. 1. Multiple data set registration of two femur bone fractures to the whole bone model (template). T1, T2 and T12 are the rigid transformations mapping Fracture 1 to the bone template, Fracture 2 to the bone template and Fractures 2 and 1 to each other, respectively.

The last point is to distinct the pair-wise registration from the global registration algorithms. The former registers only two data sets at a time; however, the global registration techniques simultaneously register multiple data sets. The latter means that, as shown in Figure 1 all the overlapping areas can be simultaneously considered in the global registration algorithms to estimate the transformation parameters. One may argue that pair-wise registration algorithms may also be utilized to solve the global registration problem for multiple data sets, by sequentially registering each two pairs of data sets. However, this will lead to an uneven spread of registration error among the data sets [1].

In our earlier work, the Unscented Kalman Filter (UKF) algorithm was proposed for pair-wise, point-based registration 2. In this paper, we use the UKF algorithm to globally register multiple data sets, and to simultaneously estimate the transformation parameters. Two algorithms are developed for scenarios where point correspondences are known, and where the correspondences are not available; but, the data sets are roughly aligned together such that the closest point metric [3] can be used to get a reliable estimate of the correspondences. Both algorithms are verified on simulated and real data sets.

\section{Mathematical Problem Definition and Previous Work}

To mathematically define the global registration problem based on the known correspondences, let's assume there are $M$ data sets $\left(S^{1}, \ldots\right.$, and $\left.S^{M}\right)$ that might have overlap with each other. The problem is to determine $M$ rigid transformations $\left(\mathbf{T}^{1}, \mathbf{T}^{2}, \ldots\right.$, and $\left.\mathbf{T}^{M}\right)$ such that the cost function $C$ is minimized:

$$
C\left(\mathbf{T}^{1}, \ldots, \mathbf{T}^{M}\right)=\sum_{\alpha=1 \beta}^{M-1} \sum_{\beta=\alpha+1}^{M} \sum_{k=1}^{N^{\alpha, \beta}}\left(\mathbf{T}^{\alpha} \mathbf{p}_{k}^{\alpha, \underline{\beta}} \mathbf{T}^{\beta} \mathbf{p}_{k}^{\beta, \alpha}\right)^{T}\left[\boldsymbol{\Lambda}_{k}^{\alpha, \beta}\right]^{-1}\left(\mathbf{T}^{\alpha} \mathbf{p}_{k}^{\alpha, \beta} \mathbf{T}^{\beta} \mathbf{p}_{k}^{\beta, \alpha}\right),
$$

where the weight matrix $\boldsymbol{\Lambda}_{k}^{\alpha, \beta}$ is given and it models the variance of the point localization error; $\mathbf{p}_{k}^{\alpha, \beta}$ and $\mathbf{p}_{k}^{\beta, \alpha}$ are the corresponding points between $S^{\alpha}$ and $S^{\beta}$ 
data sets, respectively, and $N^{\alpha, \beta}$ is the number of corresponding points between the two data sets. It should be noted that this problem is degenerate since if the same transformation applies to all the data sets, it causes no difference in the registration. This degeneracy can be removed by assuming that one of the transformations, such as $\mathbf{T}^{1}$, to be identity. Therefore, there are $M-1$ transformations remaining that should be estimated.

Thus far, many global registration algorithms have been proposed to solve the multiple data set registration problem using the gradient descent technique [4, "mean shape" [5], Quaternions [6], the Expectation Maximization (EM) algorithm [7, the classic Gauss method [8], and the first order kinematics [9]. All these algorithms iteratively minimize the cost function $C$, by considering all the corresponding points at once. Instead, we propose an iterative minimization approach, using the Unscented Kalman Filter (UKF) algorithm, to simultaneously estimate the transformation parameters by incrementally (one by one) feeding the corresponding points to the registration algorithm. Since the optimization method usually converges well before all the corresponding points are used in the registration process, the algorithm is computationally efficient and can potentially make real-time clinical applications feasible. Furthermore, the proposed approach can compute the variance of the estimated transformation parameters, which could be used as a measure for the registration accuracy.

\section{Method}

Let us assume there are $M-1$ rigid transformations $\left(\mathbf{T}^{2}, \ldots\right.$, and $\left.\mathbf{T}^{M}\right)$ to be estimated from $M$ potentially overlapping data sets. Data set one is assumed to be in the reference frame $\left(\mathbf{T}^{1}=\mathbf{I}_{3 \times 3}\right)$. Each rigid transformation $\mathbf{T}^{\alpha}$ consists of a rotation matrix $\mathbf{R}^{\alpha}\left(\theta_{x}^{\alpha}, \theta_{y}^{\alpha}, \theta_{z}^{\alpha}\right.$ ) (The Euler angles are used to represent the rotation matrix.), and a translation vector $\mathbf{t}^{\alpha}=\left[t_{x}^{\alpha}, t_{y}^{\alpha}, t_{z}^{\alpha}\right]$. Therefore, $(M-1) \times 6$ parameters are needed to estimate $M-1$ rigid transformations. Let's define the state vector $\mathbf{x}$ comprising these transformation parameters as:

$$
\begin{aligned}
\mathbf{x} & =\left[t_{x}^{2}, t_{y}^{2}, t_{z}^{2}, \theta_{x}^{2}, \theta_{y}^{2}, \theta_{z}^{2}, \ldots, t_{x}^{M}, t_{y}^{M}, t_{z}^{M}, \theta_{x}^{M}, \theta_{y}^{M}, \theta_{z}^{M}\right]^{T}, \\
& =\left[\mathbf{x}_{t}^{2^{T}}, \mathbf{x}_{\theta}^{2^{T}}, \ldots, \mathbf{x}_{t}^{M^{T}}, \mathbf{x}_{\theta}^{M^{T}}\right]^{T},
\end{aligned}
$$

where $\mathbf{x}_{t}^{\alpha}=\left[t_{x}^{\alpha}, t_{y}^{\alpha}, t_{z}^{\alpha}\right]^{T}$ and $\mathbf{x}_{\theta}^{\alpha}=\left[\theta_{x}^{\alpha}, \theta_{y}^{\alpha}, \theta_{z}^{\alpha}\right]^{T}$. The process model is assumed to be governed by a linear function as:

$$
\mathbf{x}_{k}=\mathbf{x}_{k-1}+\mathcal{N}\left(0, \mathbf{\Sigma}_{Q}\right),
$$

where $\mathbf{x}_{k}$ is the defined state vector at time $k$ (when the $k$ th pair of corresponding points is fed into the algorithm) with the initial value and covariance matrix $\mathbf{x}_{0}$ and $\mathbf{P}_{x}^{0}$, respectively. $\mathcal{N}\left(0, \boldsymbol{\Sigma}_{Q}\right)$ is a zero-mean, Gaussian random vector with covariance matrix $\boldsymbol{\Sigma}_{Q}$. The goal is to incrementally estimate the state vector $\mathbf{x}_{k}$ from the observation model:

$$
\mathbf{y}_{1: k}=\left\{\begin{array}{l}
\mathbf{p}_{1: k}^{1, \beta}=\mathbf{R}_{\left(\mathbf{x}_{\theta}^{\beta}\right)} \mathbf{p}_{1: k}^{\beta, 1}+\overline{\mathbf{t}}_{\left(\mathbf{x}_{\mathbf{t}}^{\beta}\right)}+\mathcal{N}\left(0, \boldsymbol{\Lambda}_{1: k}^{1, \beta}\right), \alpha=1, \\
\mathbf{0}=\mathbf{R}_{\left(\mathbf{x}_{\theta}^{\alpha}\right)} \mathbf{p}_{1: k}^{\alpha, \beta}+\overline{\mathbf{t}}_{\left(\mathbf{x}_{\mathbf{t}}^{\alpha}\right)}-\mathbf{R}_{\left(\mathbf{x}_{\theta}^{\beta}\right)} \mathbf{p}_{1: k}^{\beta, \alpha}-\overline{\mathbf{t}}_{\left(\mathbf{x}_{\mathbf{t}}^{\beta}\right)}+\mathcal{N}\left(0, \boldsymbol{\Lambda}_{1: k}^{\alpha, \beta}\right), \alpha=2, \ldots, M-1,
\end{array}\right.
$$


where $\beta=\alpha+1, \ldots, M, \mathbf{p}_{1: k}^{\alpha, \beta}=\left[\mathbf{p}_{1}^{\alpha, \beta}, \ldots, \mathbf{p}_{k}^{\alpha, \beta}\right], \overline{\mathbf{t}}_{\left(\mathbf{x}_{\mathrm{t}}^{\alpha}\right)}=\left[\mathbf{t}_{\left(\mathbf{x}_{\mathbf{t}}^{\alpha}\right)}, \ldots, \mathbf{t}_{\left(\mathbf{x}_{\mathbf{t}}^{\alpha}\right)}\right]_{3 \times k}$, and $\mathcal{N}\left(0, \boldsymbol{\Lambda}_{1: k}^{\alpha, \beta}\right)$ is a zero-mean, Gaussian random vector with covariance matrix $\boldsymbol{\Lambda}_{1: k}^{\alpha, \beta}=\operatorname{diag}\left(\boldsymbol{\Lambda}_{1}^{\alpha, \beta}, \ldots, \boldsymbol{\Lambda}_{k}^{\alpha, \beta}\right)$, that models the point localization error in data sets, and $\mathbf{y}_{1: k}$ is the column vector with the maximum size of $[3 k M(M-1) / 2] \times 1$. To estimate the state vector $\mathbf{x}$, the following algorithm iterates until it converges to the final solution: 1) Predict the state vector $\mathbf{x}$ and its covariance matrix $\mathbf{P}_{\mathbf{x}}$ from the state model, Equation (3), as: $\hat{\mathbf{x}}_{k}^{-}=\hat{\mathbf{x}}_{k-1}$, and $\mathbf{P}_{\hat{\mathbf{x}}_{k}^{-}}=\mathbf{P}_{\hat{\mathbf{x}}_{k-1}}+\mathbf{\Sigma}_{Q}$, respectively; 2) Append the $k$ th pair of corresponding points from the overlapping data sets to the set of already selected pairs, and predict the corresponding points' positions in the reference frame using the computed state vector in Step 1:

$$
\hat{\mathbf{y}}_{1: k}=\left\{\begin{array}{l}
\mathbf{R}_{\left(\hat{\mathbf{x}}_{\theta}^{\beta}\right)} \mathbf{p}_{1: k}^{\beta, 1}+\overline{\mathbf{t}}_{\left(\hat{\mathbf{x}}_{\mathrm{t}}^{\beta}\right)}, \\
\mathbf{R}_{\left(\hat{\mathbf{x}}_{\theta}^{\alpha}\right)} \mathbf{p}_{1: k}^{\alpha, \beta}+\overline{\mathbf{t}}_{\left(\hat{\mathbf{x}}_{\mathbf{t}}^{\alpha}\right)}-\mathbf{R}_{\left(\hat{\mathbf{x}}_{\theta}^{\beta}\right)} \mathbf{p}_{1: k}^{\beta, \alpha}-\overline{\mathbf{t}}_{\left(\hat{\mathbf{x}}_{\mathbf{t}}^{\beta}\right)},
\end{array}\right.
$$

; 3) Calculate the distance error of the corresponding points in the reference frame to update the state vector and its covariance matrix as:

$$
\hat{\mathbf{x}}_{k}=\hat{\mathbf{x}}_{k}^{-}+\mathbf{K}_{k}\left(\mathbf{y}_{1: k}-\hat{\mathbf{y}}_{1: k}\right), \quad \mathbf{P}_{\hat{\mathbf{x}}_{k}}=\mathbf{P}_{\hat{\mathbf{x}}_{k}^{-}}-\mathbf{K}_{k} E\left[\mathbf{y}_{1: k} \mathbf{y}_{1: k}^{T}\right] \mathbf{K}_{k}^{T},
$$

where $\mathbf{K}_{k}=E\left[\mathbf{x}_{k} \mathbf{y}_{1: k}^{T}\right] / E\left[\mathbf{y}_{1: k} \mathbf{y}_{1: k}^{T}\right]$ is called the Kalman gain. This procedure iterates through all pairs of corresponding points until convergence to a solution.

For the case where correspondences among data sets are not available, the proposed algorithm can be extended to use the closest point metric [3] to attain the correspondences. Here, it is assumed that the data sets are roughly aligned such that the closest point metric returns reliable candidates of the corresponding points to initiate the optimization process. Then, the correspondences are updated while the data sets are being registered to each other using the following algorithm: 1) Choose three points from each data set and find their corresponding points in the overlapping areas in the other data sets; 2) Use the correspondences and the proposed UKF registration algorithm to register the data sets; 3) Apply the estimated transformations to the data sets; 4) Append another point from each data set to the set of points already selected from that data set. Update the location of the corresponding points (using the closest point metric) for all the points considered in the optimization until this iteration, and go to Step 2. This procedure iterates till it converges to a solution.

\section{Results}

To verify the proposed algorithm, three simulations were performed in MAT$\mathrm{LAB}$ on a $2 \mathrm{GHz} \mathrm{PC}$ with $512 \mathrm{MB}$ of RAM. We first performed an experiment on randomly generated data sets based on given correspondences. To do so, three overlapping data sets in the range of $\pm 100 \mathrm{~mm}$ were generated. Every data set consisted of 100 points, each with 50 overlapping points. Two rigid random transformations, in the range of $\left[ \pm 50^{\circ}, \pm 50 \mathrm{~mm}\right]$, were applied to two of the data sets to bring them out of alignment. Three zero-mean, Gaussian noise distributions with different variances were added to three overlapping areas among the 

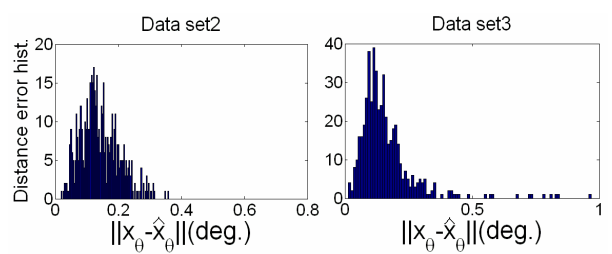

Table 1. Surface registration error using the proposed and Pennec's algorithms over 500 trials for three different experiments (Ov.:overlapping area)
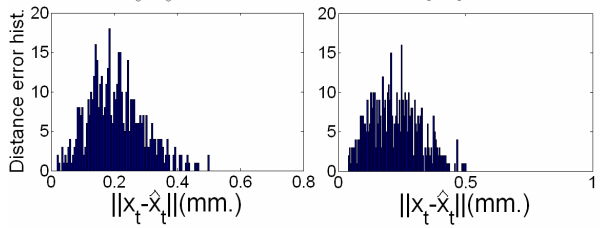

\begin{tabular}{|c|c|c|c|}
\hline \multirow{2}{*}{$\begin{array}{c}\text { Three } \\
\text { Exps. }\end{array}$} & \multicolumn{3}{|c|}{ Mean square surf. reg. error } \\
\cline { 2 - 4 } & Ov.1\&2 & Ov.1\&3 & Ov.2\&3 \\
\hline \hline Var. noise & $1 \mathrm{~mm}^{2}$ & $1 \mathrm{~mm}^{2}$ & $1 \mathrm{~mm}^{2}$ \\
\hline Pennec's & $0.96 \mathrm{~mm}^{2}$ & $0.98 \mathrm{~mm}^{2}$ & $1 \mathrm{~mm}^{2}$ \\
UKF & $0.97 \mathrm{~mm}^{2}$ & $0.99 \mathrm{~mm}^{2}$ & $0.97 \mathrm{~mm}^{2}$ \\
\hline \hline Var. noise & $3 \mathrm{~mm}^{2}$ & $2 \mathrm{~mm}^{2}$ & $0.025 \mathrm{~mm}^{2}$ \\
\hline Pennec's & $2.91 \mathrm{~mm}^{2}$ & $1.93 \mathrm{~mm}^{2}$ & $0.14 \mathrm{~mm}^{2}$ \\
UKF & $2.97 \mathrm{~mm}^{2}$ & $1.95 \mathrm{~mm}^{2}$ & $0.027 \mathrm{~mm}^{2}$ \\
\hline \hline Var. noise & $3 \mathrm{~mm}^{2}$ & $2 \mathrm{~mm}^{2}$ & $0.001 \mathrm{~mm}^{2}$ \\
\hline Pennec's & $2.9 \mathrm{~mm}^{2}$ & $1.92 \mathrm{~mm}^{2}$ & $0.12 \mathrm{~mm}^{2}$ \\
UKF & $2.96 \mathrm{~mm}^{2}$ & $1.95 \mathrm{~mm}^{2}$ & $0.004 \mathrm{~mm}^{2}$ \\
\hline
\end{tabular}

Fig. 2. Distribution of the estimated transformation parameters, using the proposed algorithm, over 500 trials

data sets. Then, the proposed algorithm was used to register the two data sets back to their original places. The procedure was repeated for 500 trials. On average, in each trial it took 3 seconds to finalize the registration. We used different noise levels added to data sets to verify the performance of the algorithm. We also compared the performance of the proposed technique with the one reported by Pennec [5]. Table 1 displays the variances considered for each overlapping area among the data sets, and the mean square surface registration error after global registration of the data sets. As shown, the proposed algorithm more accurately registers the data sets than the Pennec's algorithm since the mean squared surface registration error calculated by the proposed algorithm is closer to the variance of the noise perturbing the data sets. Figure 2 depicts the distance error histograms of the rotation and translation parameters for one of the trials where the variance of the noise added to each overlapping area was $1 \mathrm{~mm}^{2}$. Furthermore, in this simulation, in order to show the superiority of the proposed global registration algorithm over the sequential registration algorithms, we sequentially registered data sets 2 and 3 to data set 1 . In this case, the mean squared surface registration errors between data sets 2 and 3 were calculated as $1.1 \mathrm{~mm}^{2}, 0.3 \mathrm{~mm}^{2}$, and $0.3 \mathrm{~mm}^{2}$, respectively, for different variances of the noise (as above) perturbing that region. As expected, these variances are farther away from the ones reported by the global registration technique, demonstrating the better accuracy of the proposed technique.

In the next experiment, three data sets collected from a fractured femur bone phantom were used. The first data set was generated by taking CT images of the femur bone, before it was fractured. CT images were captured using a LightSpeed Plus CT scanner. Then, Mesher software developed at our institution was used to semi-automatically segment the CT images, and to create a 3D surface 


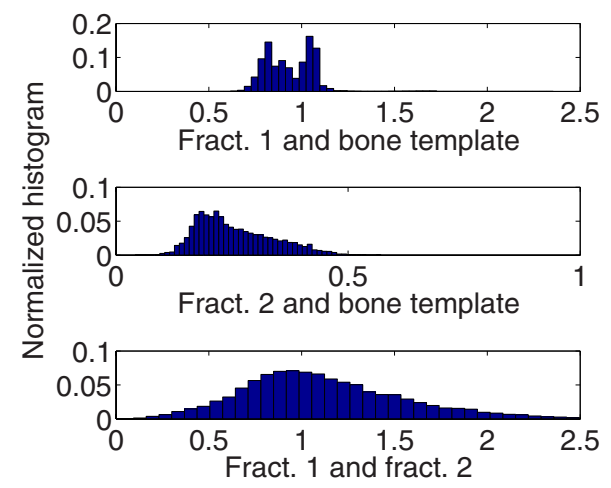

Fig. 3. Normalized histogram of surface registration error among the registered areas for femur fractures in $(\mathrm{mm})$ over 250 trials where the range of generated random transformations is $\left[ \pm 2.5^{\circ}, \pm 2.5 \mathrm{~mm}\right]$
Table 2. Convergence rate of the proposed algorithm in globally registering multiple femur bone fractures to the bone template (Fracture 1 to the template, Fracture 2 to the template and Fracture 2 to 1 ) over 250 trails for different range of random transformations; when there is no outlier and when there is outlier in the data sets

\begin{tabular}{|c|c|c|c|c|c|c|}
\hline Range of & \multicolumn{5}{|c|}{ Convergence rate in \% } \\
\cline { 2 - 7 } $\begin{array}{c}\text { Trans. } \\
\text { (mm.,deg. })\end{array}$ & 1 & 2 & $1 \& 2$ & 1 & 2 & $1 \& 2$ \\
\hline \hline \pm 2.5 & 99 & 100 & 99 & 100 & 100 & 100 \\
\pm 5 & 97 & 100 & 96 & 100 & 100 & 100 \\
\pm 10 & 82 & 97 & 87 & 98 & 98 & 98 \\
\pm 15 & 60 & 93 & 63 & 97 & 92 & 89 \\
\pm 20 & 48 & 86 & 46 & 90 & 84 & 76 \\
\hline
\end{tabular}

model (bone template) - containing 36, 508 points- using a marching cubes algorithm. To produce the other two data sets, the femur bone phantom was fractured into two pieces. Another set of CT images was taken from each piece and a 3D surface model was then generated from that piece. The generated mesh of the bone and its fractures are shown in Figure 1 Before fracturing the femur bone phantom, some fiducials were implanted on the bone surface. Those fiducials were used to register the bone fracture mesh data sets to the bone template. Two random transformations are applied on the two fracture data sets to take them out of alignment. Then, the proposed UKF-based global registration algorithm, based on unknown correspondences, was used to globally register the transformed fracture data sets back to the bone template. To do so, 100 points were randomly chosen from each overlapping area among fractures and the bone template. Those points were then used to globally register the fracture data sets back to their original locations for two cases; where there are no outliers among the fracture data sets (100 points of each fracture are registered to 100 points of the other fracture or the bone template), and where there were outliers among data sets (100 points of each fracture are registered to all the points of the other fracture or bone template). After performing registration, the rest of the fracture points which were not employed in the registration algorithm, were used to compute the surface registration error. This procedure was repeated for 250 trials for different ranges of random transformations. On average, each trial took 29 seconds to complete. Table 2 displays the convergence rate of the proposed algorithm in different simulation runs with different ranges of transformations. It is assumed that the algorithm is able to converge for registration of each overlapping area when the mean surface registration error for that area is less than $\sqrt{2.5} \mathrm{~mm}$. Figure 3 shows normalized histogram of surface registration error (in $\mathrm{mm}$ ) for the three overlapping areas after performing registration over 250 trials 


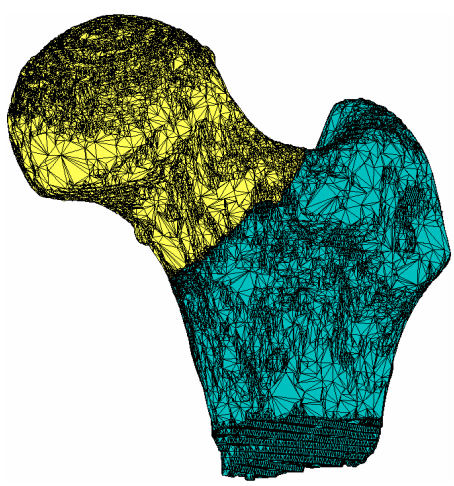

Fig. 4. Global registration of the femur bone fractures

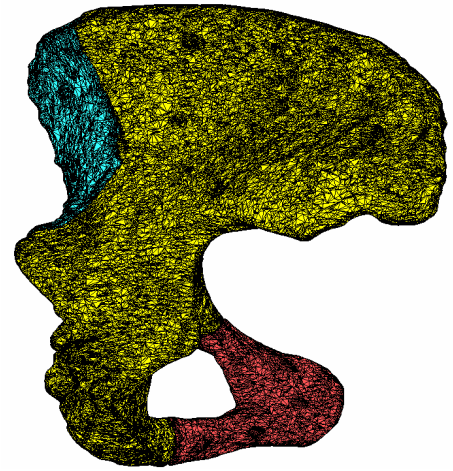

Fig. 5. Global registration of the plevis bone fractures

for the range of transformation $\left[ \pm 2.5^{\circ}, \pm 2.5 \mathrm{~mm}\right]$. Figure 4 depicts the registered fractured bone data sets to the bone template using the proposed algorithm for one of the simulation runs. In the last simulation, the above experiment was repeated on a pelvis bone phantom. Using CT images, a 3D mesh was generated from the phantom, containing 83,562 points. Then, the bone was fractured into three pieces. CT images were used to generate a surface mesh for each fractured piece. Before fracturing the phantom, some fiducials were mounted on the bone. Those markers were used to register the fractures back to their original locations on the bone template. Three random transformations in the range of $\left[ \pm 10^{\circ}, \pm 10 \mathrm{~mm}\right]$ were applied to the fractures to bring them out of alignment. Then, the proposed algorithm was used to simultaneously register 80 points, randomly selected from each fractured surface, to the bone template. This procedure was repeated for 250 trials. On average, each trial took 22 seconds to complete. Figure 5 displays the registered pelvis bone fractures to the pelvis bone template for a simulation run. Before and after performing registration, the mean surface registration error for all the overlapping areas over 250 trials were calculated to be $14.61 \mathrm{~mm}$ and $1.41 \mathrm{~mm}$, respectively (with outliers); and $14.6 \mathrm{~mm}$ and $0.83 \mathrm{~mm}$, respectively (with no outliers).

\section{Discussions and Conclusion}

The goal of this work was to present a new global registration algorithm for registering multiple overlapping data sets based on known and unknown correspondences. In the case of known correspondences, the proposed algorithm was used to globally register randomly generated data sets, and its performance was compared with the Pennec's algorithm. As shown in Table 1, the proposed algorithm was able to reduce the mean square surface registration error among the overlapping areas to the level of variance of the noise added to each data set, and was more accurate than the Pennec's technique. Where correspondences 
among data sets were not available, the closest point metric was utilized to get a reliable estimate of the correspondences. Results on fractured femur and pelvis bone phantoms, with or without outliers in the registration process, show that the algorithm is robust and can converge even when relatively large initial alignment errors exist. As future work, it would be of interest to increase the robustness of the technique to initial alignment errors. One solution would be a feature matching technique, such as robust point matching algorithm (RPM) [10], to automatically find at least three pairs of correspondences between each data set. Furthermore, We would like to compare the proposed registration algorithm with the sequential ICP registration algorithms existing in the literature. Extending the proposed algorithm for registering multiple data sets perturbed by anisotropic Gaussian noise is another issue to be investigated.

\section{Acknowledgment}

We would like to thank Dr. Maarten Beek for assisting us with CT data collection and 3D surface mesh generation of the femur and pelvic bone phantoms.

\section{References}

1. Sharp, G., Lee, S., Wehe, D.: Multiview Registration of 3D Scenes by Minimizing Error between Coordinate Frames. PAMI, 1037-1050 (2004)

2. Moghari, M., Abolmaesumi, P.: Point-based rigid-body registration using an unscented kalman filter. IEEE Trans. Medical Imaging (to appear)

3. Besl, P., McKay, H.: A Method for Registration of 3D Shapes. PAMI 14(2), 239-256 (1992)

4. Stoddart, A., Hilton, A.: Registration of Multiple Point Sets. In: ICPR, vol. 2 (1996)

5. Pennec, X.: Multiple Registration and Mean Rigid Shapes - Application to the 3D Case. Image Fusion and Shape Variability Techniques, 178-185 (1996)

6. Benjemaa, R., Schmitt, F.: A Solution for the Registration of Multiple 3D Point Sets using Unit Quaternions. In: Proc. Eur. Conf. Comp. Vis., pp. 34-50 (1998)

7. Goldberger, J.: Registration of Multiple Point Sets using the EM Algorithm. Comp. Vis. 2, 730-736 (1999)

8. Williams, J., Bennamoun, M.: A Multiple View 3D Registration Algorithm with Statistical Error Modeling. Inf. Sys., 1662-1670 (2000)

9. Pottmann, H., Leopoldseder, S., Hofer, M.: Simultaneous Registration of Multiple Views of a 3D Object. Photo. Remote Sens. Spatial Inf. Sci., 265-270 (2002)

10. Rangarajan, A., Chui, H., Bookstein, F.L.: The softassign procrustes matching algorithm. In: Duncan, J.S., Gindi, G. (eds.) IPMI 1997. LNCS, vol. 1230, pp. 29-42. Springer, Heidelberg (1997) 